\title{
Photonic Liquid Crystal Fibers - 15 years of research activities at the Warsaw University of Technology
}

\author{
T. R. Woliński, S. Ertman, K. Rutkowska, D. Budaszewski, M. Sala-Tefelska, M. Chychłowski, K. Orzechowski, \\ K. Bednarska, and P. Lesiak
}

Faculty of Physics, Warsaw University of Technology, Koszykowa 75, 00-662 Warszawa, Poland

Received June 14, 2019; accepted June 29, 2019; published June 30, 2019

\begin{abstract}
Research activities in the area of photonic liquid crystal fibers carried out over the last 15 years at the Warsaw University of Technology (WUT) have been reviewed and current research directions presented, which include metallic nanoparticles doping to enhance the electro-optical properties of photonic liquid crystal fibers.
\end{abstract}

Over the last years it has been shown that solid-core photonic crystal fibers (PCFs) can offer an additional level of tunability by infiltrating air-channels of their cladding with a tunable material. A concept of liquidcrystal (LC) inclusions resulted in a new type of an "active" optical fiber.

Since 2004, an LC-infiltrated PCF has been referred by us as a photonic liquid crystal fiber (PLCF) [1], see Fig. 1. In literature, there are also known other names: a liquid crystal-photonic crystal fiber (LC-PCF) [2], or a liquid crystal-photonic bandgap fiber [3]. High tunability of PLCFs achieved by applying external factors such as temperature, strain, pressure, electric and magnetic fields, as well as by the light beam itself (when nonlinear effects in LCs are considered), enable various potential applications in the field of fiber-optic sensing and photonic devices.

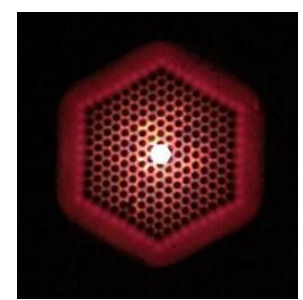

Fig. 1. A typical end face of the PLCF composed of a PCF (UMCS 061215P2) infiltrated with a nematic LC (MUT 6CHBT) observed under a digital microscope.

Infiltrating the air-holes with different LC phases allows for creation of a special class of infused PCFs with a set of improved optical properties resulting in highly-tunable photonic structures [3-4], in which light propagation conditions are determined by both an LC "guest" material and a PCF "host" structure. While the properties of effective PLCF structures strongly depend on LC molecular orientation, the techniques for efficient orientation of an LC within PCF holes were developed [5]. Hence PLCFs benefiting from the merger of passive PCF host structures with "active" LC guests are responsible for a set of unusual propagation, spectral, and polarization properties.

Light guiding dynamics, including switching between both mechanisms of propagation, can be simply achieved in PLCFs thanks to the unique properties of an infiltrating LC. Due to high electro-, magneto-, and thermo-optic responses of LCs, their refractive indices may be relatively easily changed either by temperature or external physical fields. In this context, thermal and electrical tuning along with unusual spectral and polarization properties of PLCFs have been studied in detail over the last years [6-13].

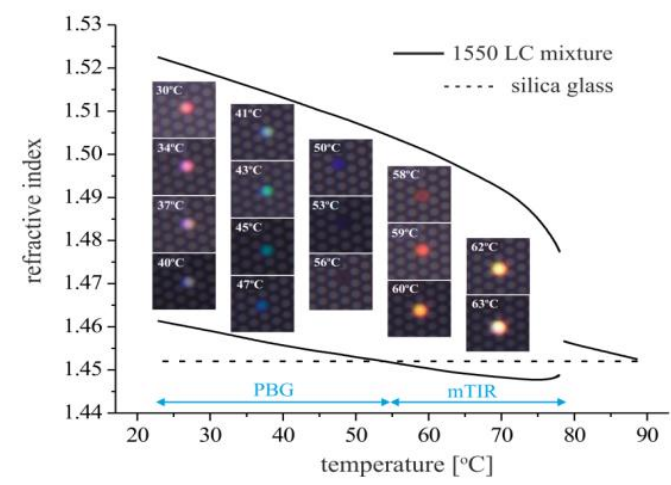

Fig. 2. Temperature-induced reversible switching between PBG and "index guiding") in a PCF filled with a low-birefringence LC mixture (1550) characterized by an ordinary refractive index higher than that of silica glass at lower temperature (PBG tuning); for higher temperatures the guiding mechanism changes to index guiding [7].

Optical wave-guiding in a PCF is governed by one of two principal mechanisms responsible for light trapping within the core. While the first one is a classical propagation effect based on the so called "index guiding" phenomenon, which is well known and similar to the wave guiding effect within a conventional fiber, the second one, referred to as the photonic band gap (PBG) effect, occurs if the refractive index of the core is lower 
than the mean reflective index of the cladding region. One of the most spectacular phenomena was the successful realization of temperature-induced switching between these two guiding mechanisms (PBG and index guiding) [7] in the PLCF composed of 1023 PCF (UMCS, Lublin, Poland) infiltrated with a low-birefringence 1550 nematic LC (MUT, Warsaw, Poland), see Fig. 2.

\section{Polarization properties of PLCFs}

Reorientation of LC molecules with a transverse electric field, in practice, always introduces a change of polarization properties of the PLCFs. There are two main polarization effects that could be observed in the PLCFs tuned with an electric field. The first effect is a change of the phase and group birefringence of the fiber. It occurs always when the LC molecules are reoriented and, as consequence, some axially symmetric change of refractive index distribution is obtained (even if the reorientation is not uniform in different micro-holes). The birefringence axis is, of course, defined by the direction of an electric field.

The second polarization effect that can be observed in the PLCFs is the introduction and tuning of polarization dependent losses (PDL). There are two main origins of asymmetry in attenuation of the two orthogonal modes: asymmetrical scattering of light by the reoriented LC molecules and particularly/predominantly change of the contrast of core-cladding refractive indices, which can lead to a drastic change of the guiding mechanism. In particular, it is possible to suppress the propagation of one of polarizations, so an isotropic fiber can be dynamically transformed into a single-polarization fiber. The axis of the PDL is, similarly as birefringence, defined by the direction of an electric field. Usually PLCFs are tuned with two flat electrodes, so only one direction of an electric field is possible. However, it is also possible to use multi-electrode steering (i.e. [14]), which, in practice, can allow for an almost arbitrary change of the direction of the field and, in consequence, a controlled change of the induced birefringence and/or PDL. By combining those effects, it is possible to build tunable wave-plates, phase shifters, polarizers or even polarization controllers and dispersion compensators [15]. Potential applications of such fibers were quite promising, but after 15 years of investigations in this area (and over EUR $1.5 \mathrm{M}$ invested in R\&D projects, only at WUT) there is still no evidence of any commercial applications.

It is worth mentioning that polarization properties of PLCFs can be defined not only by the asymmetry caused by LC molecules reorientation, but also by asymmetrical filing of the PCF with LC. Selectively filled PLCFs can be designed to have unique polarization properties even without any electric field, and reorientation of molecules can, of course, tune those properties, i.e. sign of the birefringence can be reversed [16-17].

\section{Nonlinear effects in PLCFs}

The principle advantage of introducing LCs into PCF structures is their high sensitivity to external fields and factors. It is worth underlining that apart from thermally, electrically and magnetically induced modifications, it is relatively easy to obtain optically induced changes in the refractive indices of LC due to optical nonlinearity [18].

In practical applications for sensing, nonlinear optical phenomena in PLCFs may be either applied as a measurand-sensitive interaction mechanism or as an optical signal processing technique to assist in configuring an optical signal used to probe a sensor.

In particular, a new class of nonlinear effects in NLC doped with gold nanoparticles and introduced to PCF have been reported [19].

Our other works related to nonlinear effects in PLCFs have been mostly limited to theoretical analyses [20-21] and experimental tests [22-23] related to nonlinear discrete light propagation. The latter is typical of photonic structures with a refractive index modulated periodically in space in such a way that the light can be weakly guided in waveguide channels and switching between them occurs via the evanescent coupling. Contrary to other discrete photonic systems practically achieved in various materials, PLCFs do not require specialized fabrication techniques and offer extraordinary dynamic tunability. Moreover, thanks to an almost infinite variety of host structures (PCFs) and guest materials (which are liquid crystalline materials commercially available or synthesized for special customized requirements) it is possible to obtain PLCFs to be applied as waveguide matrices for discrete propagation with almost arbitrary coupling parameters and with unusual tunable features. Specifically, nonlinear effects - related to either reorientational or thermal nonlinearities in LCs - may result in significant changes in propagation characteristics in PLCFs. Considering PLCF as a matrix of mutually parallel waveguide channels (formed here by liquid crystalline material), when optical nonlinearity is considered, spatial light localization and/or delocalization can be obtained, with the final scenario dependent on the optical power level and molecular orientation of LC [22]. In special cases, a discrete spatial soliton can be obtained, paying thus the way for all-optical sensing and switching to be developed in PLCFs. In addition, as a part of experimental tests showing the influence of an EM wave on the optical properties of PLCFs, two laser beams have been launched simultaneously into PLCF. In this case, significant changes in light intensity and spatial profile of the signal beam at the output of the fiber have been observed, proving that the propagation properties of 
PLCFs for a low power probe beam can be obtained using variable power of the pump [23].

\section{PCFs with Chiral Nematic LCs and Blue Phase LCs}

In the majority of published papers, light propagation effects in PLCFs were investigated for nematic liquid crystals filling the micro-holes in fibers. In papers [24-25] a chiral nematic PW600 LC (MUT, Warsaw, Poland) was used to fill the PCFs. It is a chiral nematic i.e. a material in which the molecules are aligned in layers which are twisted around the axis and form a helical structure. The chiral LC phase is characterized by the selective Bragg reflection, optical activity and circular dichroism. PW600 LC was characterized by the selective Bragg reflection at a wavelength of $600 \mathrm{~nm}$ and a helix pitch of $0.39 \mu \mathrm{m}$. In [24-25], light propagation effects were presented in photonic crystals fibers having 3, 5, 6 and 8 rings of holes which were filled with a chiral nematic liquid crystal. It was observed that selective light propagation in PLCF under the influence of temperature was shifted towards longer wavelengths during the cooling process and towards shorter waves during the process of heating. By using an external electric field, the attenuation of selective light propagation in PLCF was also controlled. It appears that the stronger the electric field, the higher the attenuation of PLCF. Such structures can be used, for example, as optical filters and attenuators.

Blue phase liquid crystal (BPLC) has recently attracted extensive interest in optics and photonics. This is due to its three-dimensional highly chiral structure, having interesting optical and electro-optical properties. Among them we can distinguish: (i) optical isotropy and polarization insensitivity on a macroscopic scale for wavelengths outside of the resonance band [26-27], (ii) induced birefringence by an external electric field (Kerrlike effect) [28], (iii) existence of a three-dimensional photonic band gap manifesting itself in Bragg reflections of visible light [29]. Therefore, BPLC belongs to the promising materials for innovative applications not only in novel displays, but also in advanced photonic devices. Especially, BPLC-filled photonic crystal fiber (PCF) holds a great potential for sensing and tunable filter applications by applying external fields such as temperature [30-31] or electric field [32-33], simultaneously enhanced transmission properties due to occurrence of optical isotropy in BPLC. Moreover, it was revealed that by applying an external electric field, control of transmitted light intensity for particular wavelengths (photonic bandgaps) can be achieved, depending on the input polarization. The experimental results obtained are qualitatively consistent with performed simulations relating to modal analysis of BPLC-filled PCF, utilizing physical properties of PCF and refractive index of BPLC measured before [34]. However, it should be underlined that the size of microcapillaries in a glass matrix and applied alignment layers in PCF affect the orientation of the BP domains leading to a change in optical properties of a BPLC-filled photonic microstructure $[32,35]$. Moreover, the geometry of the sample for BPLC-filling is a crucial factor. The influence of alignment layers in cylindrical and rectangular geometries on BP domains can be found elsewhere in [35] and [36], respectively. To summarize, the BPLC offers new possibilities to develop advanced LC-based photonic devices.

\section{Smectic C* LCs in PCFs}

The chiral smectic C LCs (Sm C*), also called ferroelectric liquid crystals (FLCs), are considered as one of the most suitable LC materials for infiltrating the structure of PCFs. One of the advantages of FLCs is their rapid electro-optical response, which is a very important factor in designing PLCF-based optical devices. Typical response times for commonly used nematic LCs are in a range of 10-100 ms [37-38], while for FLCs these values can drop below $100 \mu \mathrm{s}$ [39]. Moreover, to provide undisturbed light propagation in PLCFs infiltrated with FLCs and fine-tuning conditions, additional aligning materials are needed. One of the techniques resulting in an excellent alignment of FLCs in PCFs is based on a photosensitive sulfonic azo-dye material SD1 [40-41]. Research activities involved a thorough investigation of the FLC molecules alignment in silica glass microcapillaries (MCs) and PCFs [42-43]. As it was shown, a good and stable FLC alignment can be obtained by the optimal value of anchoring energy which is controlled by external UV light irradiation. Furthermore, a pattern alignment of FLC molecules inside silica glass MCs was also reported [42].

Application of FLC LCs in PCFs opens a variety of opportunities for the development of modern photonics and PLCF-based optical devices, such as in-fiber polarization controllers, fast-switching spectral filters, or optically rewritable periodic fiber gratings. First demonstrators of in-fiber polarization controllers based on PLCFs doped with FLCs were reported in [43].

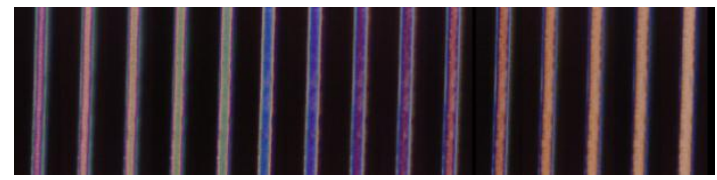

Fig. 3. Electric field-induced birefringence change in a capillary (30 $\mu \mathrm{m}$ inner diameter) filled with 6 CHBT doped with $0.1 \%$ Au NPs. The subsequent change of color is caused by electrical reorientation of the LC molecules observed under a polarizating microscope with crossed polarizers, while the capillary is at $45^{\circ}$ to polarizers axes. 


\section{Nanoparticles-doped PLCFs}

Metallic nanoparticles (NPs) were shown to exhibit interesting characteristics when coupled with a liquid crystal matrix [44]. PLCFs doped with NPs are a newly emerging type of photonic devices whose parameters can be tuned depending on the type of NPs. Among the first efforts to enhance PLCFs with NPs was doping with pure titanium (Ti). In [45] we showed that Ti NPs can modify the propagation spectra of PLCFs when exposed to temperature. There was a noticeable shift toward shorter wavelengths. Next, we investigated thermal and electrooptical properties of $4 \mathrm{~nm}$ gold ( $\mathrm{Au}$ ) NP doped PLCFs [46], see Fig. 3.

A shift towards longer wavelengths was observed along with a decrease of the nematic/isotropic phase transition temperature. Additionally, we noticed a significant decrease in threshold voltages $(\sim 50 \%)$ and shortening of rise times. In [47], similar experiments for PLCFs doped with $8 \mathrm{~nm}$ silver NPs were conducted and a $60 \%$ decrease in rise times was noticed, along with a small decrease in $\mathrm{N}-\mathrm{I}$ transition temperature. We achieved further improvement of these parameters in [48], where we doped PLCFs with $2 \mathrm{~nm} \mathrm{Au} \mathrm{NPs.} \mathrm{Up} \mathrm{to} \mathrm{80 \%} \mathrm{decrease} \mathrm{in} \mathrm{rise}$ times and up to $60 \%$ reduction in threshold voltages were observed in the samples, depending on the concentration of Au NPs. These parameters are crucial for photonic devices based on PLCFs and we believe that further improvements can be made by modifying the NPs. Experiments in this field are currently in progress and constitute a new research direction in the field of PLCFs in view of enhancing their electro-optical properties.

Support from the NCN under the grant no. UMO2015/19/B/ST7/03650 is gratefully acknowledged

\section{References}

[1] T.R. Woliński et al., Proc. SPIE, 5518, 232 (2004).

[2] F. Du, Y-Q. Lu, S.-T. Wu, Appl. Phys. Lett. 85, 2181 (2004).

[3] T.T. Larsen, A. Bjraklev, D.S. Hermann, J. Broeng, Opt. Expr. 11(20), 2589 (2003)

[4] T.R. Woliński et al., Opto-Electron. Rev. 13(2), 59 (2005).

[5] M. Chychłowski, S. Ertman, T.R. Woliński, Phot. Lett. Pol. 2(1), 31 (2010).

[6] T.R. Woliński et al., Opto-Electron. Rev. 14(4), 329(2006).

[7] T.R. Woliński et al., Meas. Sci. Technol. 17, 985 (2006).

[8] T.R. Woliński et al., IEEE Trans. Inst. Meas. 57(8), 1796 (2008).

[9] T.R. Woliński, et al., Mol. Cryst. Liq. Cryst. 502, 220 (2009).

[10] T.R. Woliński in Xiao G and Bock WJ Photonics sensing: principle and applications for safety and security monitoring (New Jersey, Wiley, 147-181, 2012)

[11] T.R. Woliński et al., Appl. Phys. A 115(2), 569 (2014).

[12] S. Ertman et al., J. Lightwave Technol. 35(16), 3399 (2017).

[13] S. Ertman et al., J. Lightwave Technol. 37(11), 2516 (2019).

[14] M.M. Tefelska et al., J. Lightwave Technol. 33(2), 2405 (2015).

[15] S. Ertman et al., J. Lightwave Technol. 30(8), 1208 (2012).

[16] K. Mileńko, S. Ertman, T.R. Woliński, Proc. SPIE 8794 (2013).

[17] O. Jaworska, S. Ertman, Phot. Lett. Poland 9(3), 79 (2017).
[18] I.C. Khoo, S.T.Wu, World Scientific (1993).

[19] P. Lesiak et al., Proc. SPIE 10228, 102280N (2017).

[20] K. Rutkowska, T. Woliński, Phot. Lett. Poland 2(3), 107 (2010).

[21] K. Rutkowska, L-W. Wei, Phot. Lett. Poland 4(4), 161 (2012).

[22] K. Rutkowska, U. Laudyn, P. Jung, Phot. Lett. Poland 5(1), 17 (2013).

[23] M. Murek, K. Rutkowska, Phot. Lett. Poland 6(2), 74 (2014).

[24] M.M. Tefelska et al., Mol. Cryst. Liq. Cryst., 558, 184 (2012).

[25] M.M. Tefelska et al., Acta Phys. Pol. A, 118, 1259 (2010).

[26] K. Orzechowski et al., Opt. Mater. 69, 259 (2017).

[27] H. Yoshida et al., Phys. Rev. E 94, 042703 (2016).

[28] J. Yan et al., Appl. Phys. Lett. 96, 071105 (2010).

[29] C.-W. Chen et al., Opt. Express 20, 23978 (2012).

[30] C.-H. Lee et al., Appl. Opt. 52, 4849 (2013).

[31] D. Poudereux et al., Proc. SPIE 92990 (2014)

[32] K. Orzechowski et al., Opt. Expr. 27, 10, 14270 (2019).

[33] M. Wahle, J. Ebel, D. Wilkes, H.S. Kitzerow, Opt. Expr. 24, 20, 22718 (2016).

[34] K. Orzechowski et al., Phot. Lett. Pol. 9(2), 54 (2017).

[35] M.M. Sala-Tefelska et al., Opt. Mater. 75, 211 (2018).

[36] M.M. Sala-Tefelska et al., Phot. Lett. Pol. 10(4), 100 (2018).

[37] P.G. de Gennes JP. The Physics of Liquid Crystals (Oxford University Press 1995).

[38] L.M. Blinov, V.G. Chigrinov, Electrooptic Effects in Liquid Crystal Materials (New York, NY: Springer New York 1994).

[39] D. Budaszewski, A.J. Srivastava, V.G. Chigrinov, T.R. Woliński, Liq. Cryst., 46(2), 272 (2019).

[40] V.G. Chigrinov, V.M. Kozenkov, H-S. Kwok. Photoalignment of Liquid Crystalline Materials (Chichester, UK: John Wiley \& Sons, Ltd 2008).

[41] M. Schadt et al., Jpn. J. Appl. Phys.31, 2155 (1992).

[42] D. Budaszewski et al., Opt. Lett. 39, 4679 (2014).

[43] D. Budaszewski, et al., J. Soc. Inf. Disp. 23, 196 (2015).

[44] O. Stamatoiu, J. Mirzaei, X. Feng, T. Hegmann, Top. Curr. Chem. 318, 331 (2012).

[45] A. Siarkowska et al., Phot. Lett. Pol. 8(1), 29 (2016).

[46] A. Siarkowska et al., Beilstein J. Nanotechnol. 8, 2790 (2017).

[47] D. Budaszewski et al., J. Mol. Liq. 267, 271 (2018).

[48] D. Budaszewski et al., Opt. Exp. 27(10), 14260 (2019). 